\title{
ORIGINAL ARTICLE HER2 induced EMT and tumorigenicity in breast epithelial progenitor cells is inhibited by coexpression of EGFR
}

\author{
S Ingthorsson ${ }^{1,2}$, K Andersen $^{3,4}$, B Hilmarsdottir ${ }^{1,2}$, GM Maelandsmo $^{3,4,5}$, MK Magnusson $^{1,2,6}$ and T Gudjonsson ${ }^{1,2}$
}

The members of the epidermal growth factor receptor (EGFR) kinase family are important players in breast morphogenesis and cancer. EGFR2/HER2 and EGFR expression have a prognostic value in certain subtypes of breast cancer such as HER2-amplified, basal-like and luminal type B. Many clinically approved small molecular inhibitors and monoclonal antibodies have been designed to target HER2, EGFR or both. There is, however, still limited knowledge on how the two receptors are expressed in normal breast epithelium, what effects they have on cellular differentiation and how they participate in neoplastic transformation. D492 is a breast epithelial cell line with stem cell properties that can undergo epithelial to mesenchyme transition (EMT), generate luminal- and myoepithelial cells and form complex branching structures in three-dimensional (3D) culture. Here, we show that overexpression of HER2 in D492 (D492 ${ }^{\text {HER2 }}$ ) resulted in EMT, loss of contact growth inhibition and increased oncogenic potential in vivo. HER2 overexpression, furthermore, inhibited endogenous EGFR expression. Re-introducing EGFR in D492 ${ }^{\text {HER2 }}$ (D492 ${ }^{\text {HER2/EGFR }}$ ) partially reversed the mesenchymal state of the cells, as an epithelial phenotype reappeared both in 3D cultures and in vivo. The D492 ${ }^{\text {HER2/EGFR }}$ xenografts grow slower than the D492 ${ }^{\mathrm{HER} 2}$ tumors, while overexpression of EGFR alone (D492 ${ }^{\mathrm{EGFR}}$ ) was not oncogenic in vivo. Consistent with the EGFR-mediated epithelial phenotype, overexpression of EGFR drove the cells toward a myoepithelial phenotype in 3D culture. The effect of two clinically approved anti-HER2 and EGFR therapies, trastuzumab and cetuximab, was tested alone and in combination on D492 $2^{\text {HER2 }}$ xenografts. While trastuzumab had a growth inhibitory effect compared with untreated control, the effect of cetuximab was limited. When administered in combination, the growth inhibitory effect of trastuzumab was less pronounced. Collectively, our data indicate that in HER2-overexpressing D492 cells, EGFR can behave as a tumor suppressor, by pushing the cells towards epithelial differentiation.

Oncogene (2016) 35, 4244-4255; doi:10.1038/onc.2015.489; published online 21 December 2015

\section{INTRODUCTION}

Carcinomas of the breast can be classified by their intrinsic gene expression pattern, and the phenotype of the various tumor subtypes has been postulated to mimic specific differentiation stages within breast development. ${ }^{1,2}$ It has been demonstrated that luminal cancers show a gene expression pattern consistent with the differentiated luminal compartment, while the HER2 (EGFR2, ErbB2) and triple negative subgroups show a more undifferentiated expression pattern. This is consistent with the tumor subgroups to arise from luminal and basal-like progenitor cells, respectively. ${ }^{3,4}$

How various critical oncogenic signals affect the differentiation state and thus affect the breast cancer subgrouping is still not well known. The reason for this is partly the lack of in vitro culture systems, modeling both normal breast stem cell biology and neoplastic transformation. D492 is a breast epithelial cell line with stem cell properties that was established by isolating and immortalizing a $\mathrm{MUC1}^{-}, \mathrm{EpCAM}^{+}$suprabasal cell population from normal primary tissue. ${ }^{5}$ D492 cells differentiate into both luminaland myoepithelial cells and form branching bi-layered cellular structures resembling the terminal duct lobular units when cultured in reconstituted basement membrane matrix (rBM). Furthermore, these cells can respond to microenvironmental signals to undergo epithelial to mesenchyme transition (EMT). ${ }^{6}$
EMT is a pivotal step during cancer progression where cells acquire motility by losing epithelial characteristics such as expression of cytokeratins and E-cadherin, and gain expression of mesenchymal markers, like vimentin, fibronectin and $\mathrm{N}$-cadherin (reviewed by Moyret-Lalle et al. ${ }^{7}$ ). Changes in microRNA (miR) expression, such as the miR-200 members, have also been implicated with EMT in breast cancer. ${ }^{8-11}$ Given phenotypic flexibility and the stem cell nature of the D492 cells, they have a unique potential to model the effect of critical signaling molecules on cellular morphogenesis, differentiation, EMT and neoplastic transformation.

Dysregulation of the signaling pathways activated by the epidermal growth factor receptor (EGFR) family is oncogenic in many epithelial cells. Amplification of the HER2 (ErbB2) gene is especially injurious in breast epithelium where upregulation of HER2 is seen in approximately $20 \%$ of all breast cancers, ${ }^{12}$ leading to increased proliferation, cell motility and metastasis. ${ }^{13}$ These receptors are also key components in controlling polarization and cellular differentiation of the breast gland (reviewed by Sternlicht et $a .^{14}$ ).

The clinical effects of HER2 and EGFR dysregulation are well known, and a range of options for inhibiting their adverse effects exists. ${ }^{15}$ New breast cancer treatment regimens have, however, actualized new clinical challenges. Development of resistance

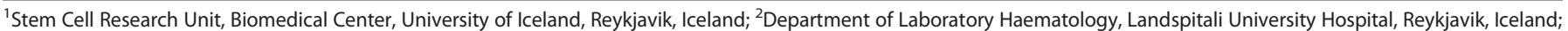

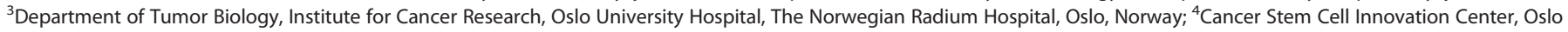

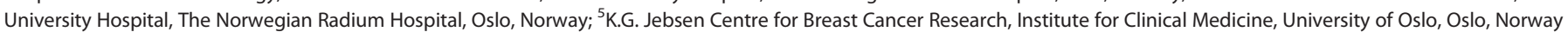

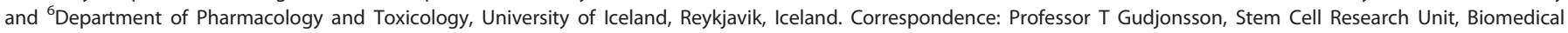
Center, University of Iceland, Vatnsmyrarvegur 16, Reykjavik 101, Iceland.

E-mail tgudjons@hi.is

Received 15 July 2015; revised 11 November 2015; accepted 14 November 2015; published online 21 December 2015
} 
against EGFR family-targeted therapy is common, and in the HER2 breast cancer subgroup, systemic disease is now partially controlled, while metastatic disease in the central nervous system seems to escape both targeted and conventional therapy. ${ }^{16}$ Increased understanding how HER2 and EGFR signaling affects cellular behavior and differentiation in breast epithelial cells is therefore needed. The objective of this study was to address the functional consequences of HER2 and EGFR overexpression in breast epithelial progenitor cells focusing on both cellular differentiation and tumorigenicity. In vitro three-dimensional (3D) models and in vivo tumorigenicity assays were employed to measure changes in cellular phenotype, stemness and tumorinitiating ability.

\section{RESULTS}

HER2 and EGFR show distinct expression pattern in human breast epithelium

Initially, we analyzed HER2 and EGFR expression in the normal breast. CK19 and CK14 were used to identify luminal epithelial- and myoepithelial cells, respectively (Figure 1, top left). Co-staining of EGFR and HER2 with either CK19 or CK14 revealed distinct expression patterns with EGFR expression associated with the basal/myoepithelial compartment. HER2 expression was predominantly associated with the luminal epithelial cells (Figure 1, lower right). Co-staining of EGFR and HER2 revealed cells within the myoepithelial compartment being positive for both receptors (Figure 1, top right). Western blotting of isolated primary luminal- and myoepithelial cells from reduction mammoplasties confirmed a higher expression of HER2 in luminal epithelial cells compared with myoepithelial cells, and more EGFR in myoepithelial cells (Figure 1b) compared with luminal cells.

Overexpression of HER2 in D492 breast epithelial progenitor cell line leads to reduced EGFR expression and EGF-independent activation of EGFR and HER2

Corresponding to the basal-like phenotype of $\mathrm{D} 492^{6,17}$ the cells express very low levels of HER2 (Figures $2 \mathrm{a}$ and b). To analyze the
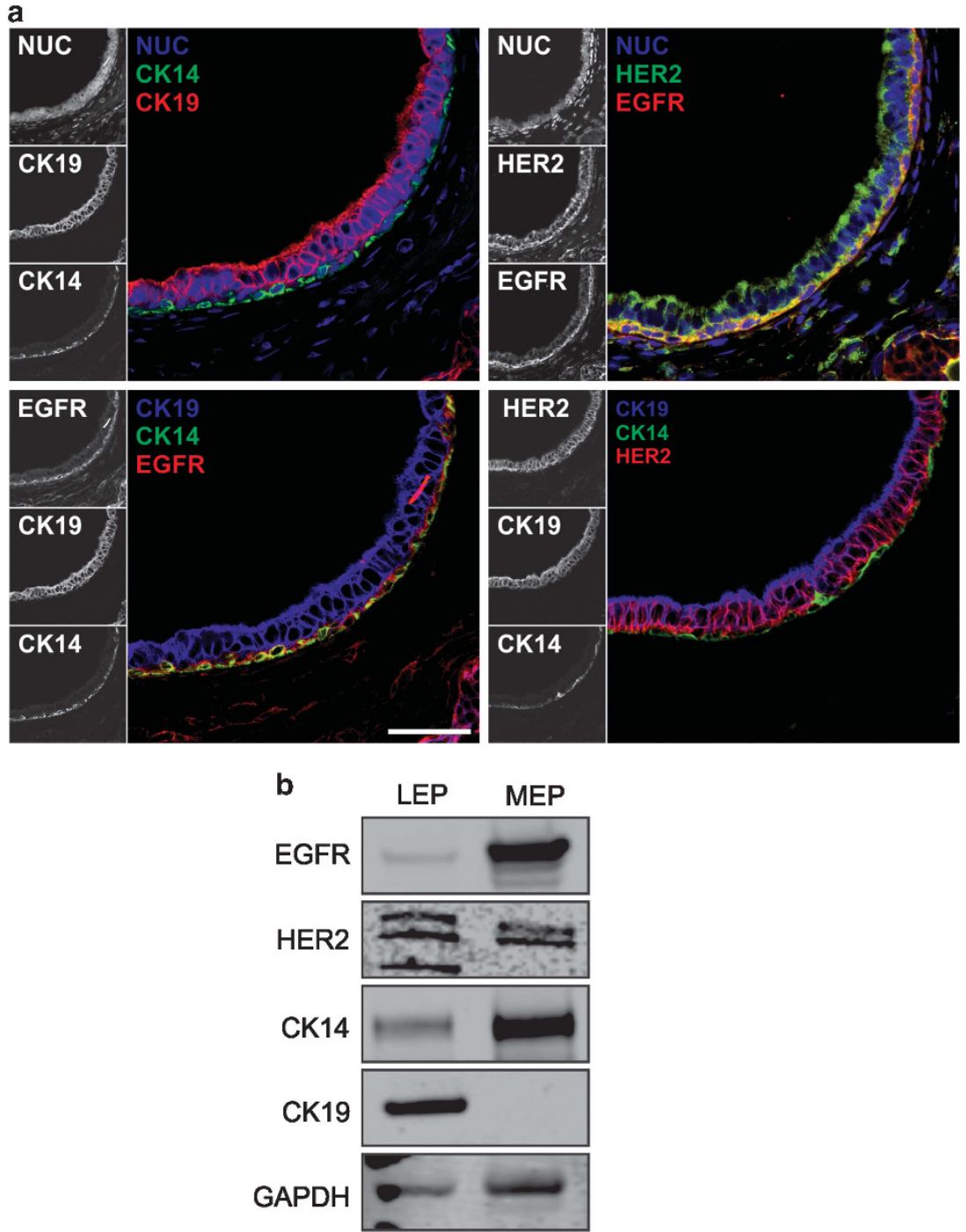

Figure 1. Expression of EGFR and HER2 in normal human breast gland. (a) HER2 and EGFR are expressed in luminal and myoepithelial cells, respectively. Confocal microscopy images of human mammary gland tissue sections. The sections were stained with antibodies against CK19, CK14, EGFR and HER2 in various combinations, and the images show part of an intralobular duct. Cytokeratin 19 and 14 (top left) identify luminal and myoepithelial cells, respectively. Bar $=50 \mu \mathrm{m}$. (b) Expression of EGFR and HER2 in cultured primary breast epithelial cells. Western blot of lysates made from purified primary myoepithelial (MEP) and luminal (LEP) epithelial cells from normal human mammary gland, stained with antibodies against EGFR, HER2, CK14 and CK19. GAPDH = loading control. 
a
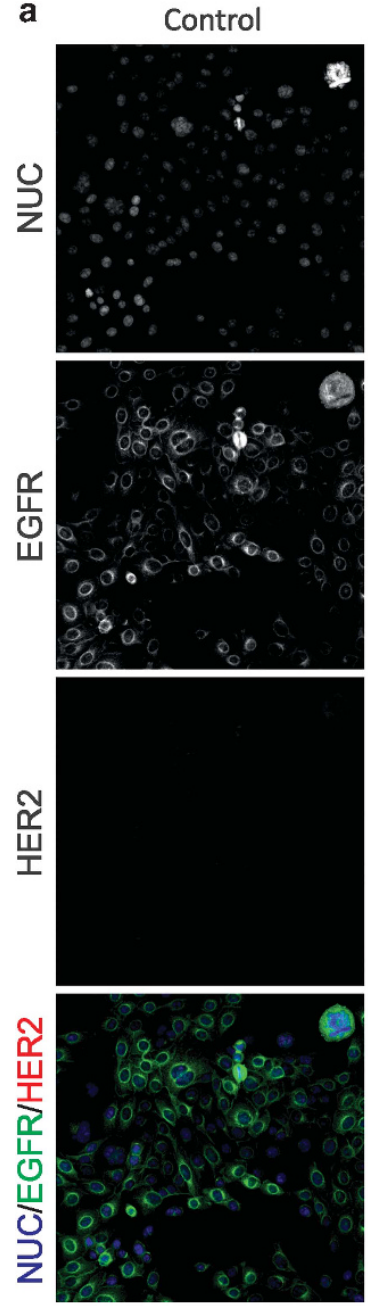

b

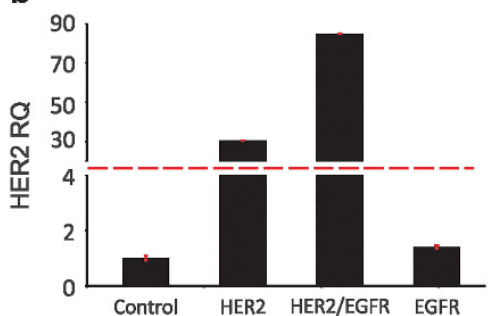

+HER2/EGFR
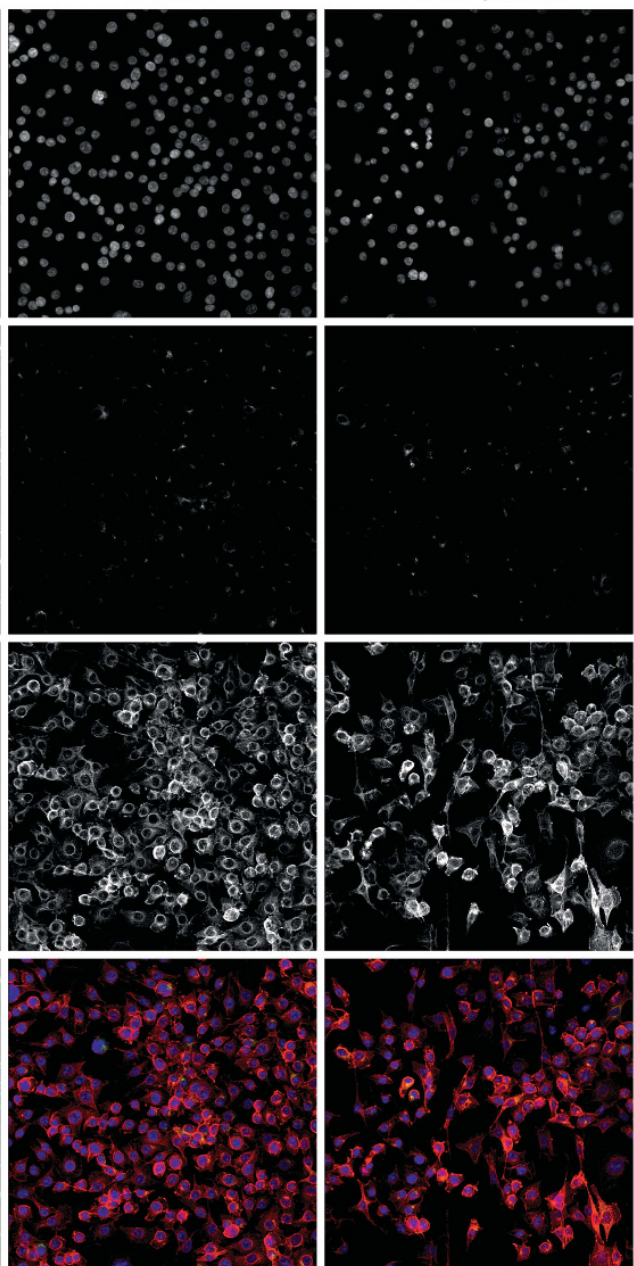
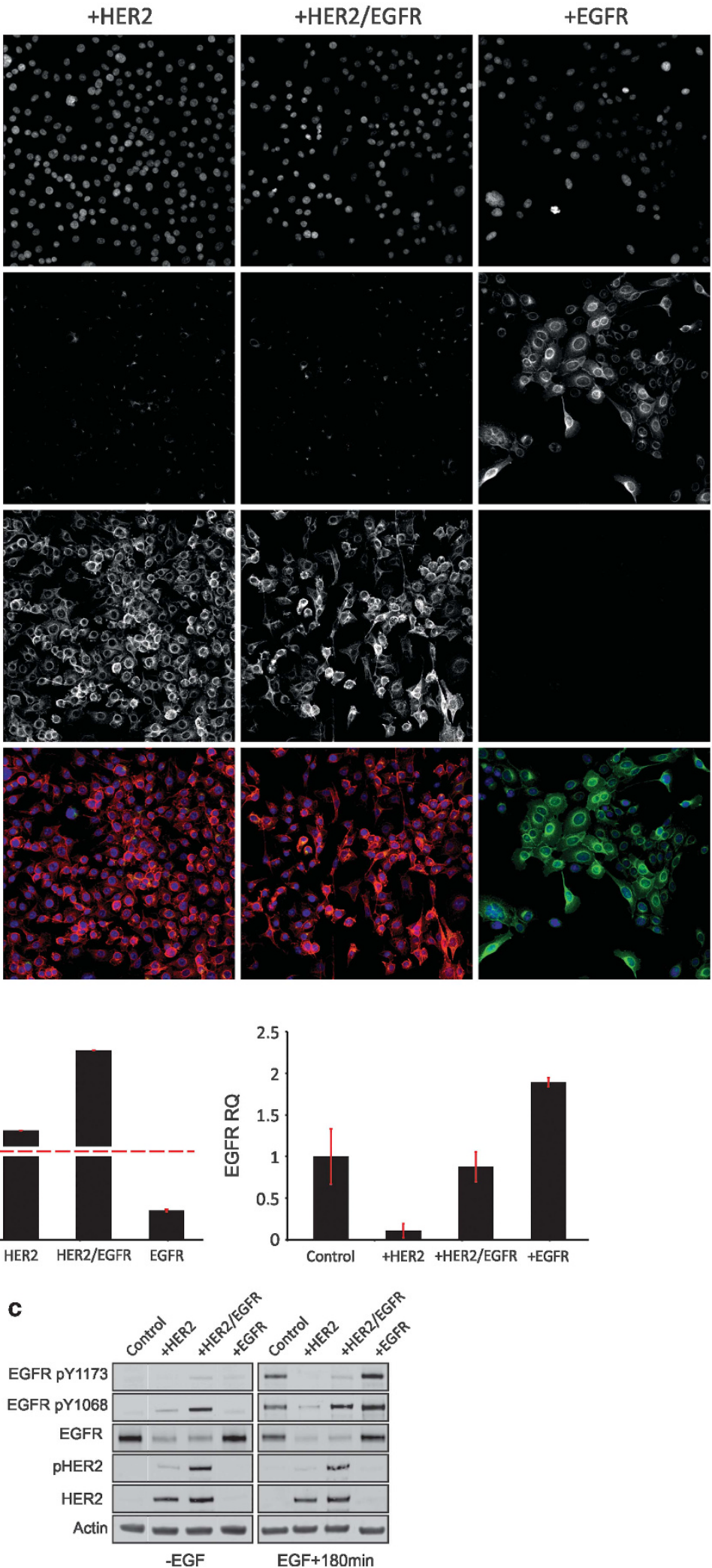

Figure 2. HER2 overexpression reduces EGFR expression. (a) Expression of endogenous EGFR in D492 ${ }^{\mathrm{HER} 2}$ is reduced compared with D492 ${ }^{\text {ctrl }}$. Confocal microscopy images of D492 cells expressing ctrl, HER2, EGFR and HER2/EGFR grown on culture flasks and analyzed by immunofluorescence staining for EGFR and HER2 expression. Bar $=50 \mu \mathrm{m}$. (b) HER2 overexpression leads to reduced EGFR transcription levels. Quantitative reverse transcriptase-PCR was performed using monolayer RNA isolates from all four cell lines. Transcription of HER2 and EGFR was analyzed, and normalized to GAPDH. (c) HER2 overexpression leads to ligand-independent EGFR and HER2 phosphorylation. Cells on monolayer were starved for $24 \mathrm{~h}$ in media without EGF. Cells were then given control media or EGF-containing culture media. Protein lysates were collected at $+180 \mathrm{~min}$ and blotted for total and phosphorylated EGFR and HER2. Actin = loading control. 
differentiation- and oncogenic potential of HER2 on mammary progenitor cells, the protein was overexpressed in D492 (Supplementary Figure S1). HER2 transduction (D492 ${ }^{\mathrm{HER} 2}$ ) resulted in high expression at both protein (Figure 2a) and transcriptional level (Figure 2b). Interestingly, endogenous EGFR expression was greatly reduced in the D492 ${ }^{\mathrm{HER} 2}$ cells; reduced staining of EGFR (Figure 2a) correlates well with reduced transcription of the EGFR gene (Figure 2b). Transduction of EGFR into D492 ${ }^{\text {HER2 }}$ (D492 ${ }^{\text {HER2/EGFR }) ~ p a r t i a l l y ~ r e s t o r e d ~ E G F R ~ e x p r e s s i o n ~(F i g u r e ~ 2 a) . ~}$ Quantitative reverse transcriptase-PCR was performed to confirm that the reduced EGFR levels was caused by transcriptional repression of EGFR mRNA (Figures $2 a$ and $b$ ).

To investigate whether overexpression of HER2 led to changes in the activation state of the two receptors, cells were starved and then treated with EGF for up to $3 \mathrm{~h}$. In the absence of EGF, no phosphorylation of either tyrosine (Y)1173 or 1068 on EGFR was detected in D492 ${ }^{\mathrm{ctrl}}$ and D492 ${ }^{\mathrm{EGFR}}$. In contrast, while strong EGFR downregulation could be seen in D492 ${ }^{\mathrm{HER} 2}$, the cells show detectable $\mathrm{EGFR}^{\mathrm{Y} 1068}$ and HER2 ${ }^{\mathrm{Y} 1221 / 1222}$ phosphorylation, demonstrating EGF-independent activation of both receptors (Figure 2c). Upon addition of EGF, strong phosphorylation at Y1173 and Y1068 was detected after 3 and $30 \mathrm{~min}$ in both D492 ${ }^{\mathrm{crl}}$ and D492 ${ }^{\mathrm{EGFR}}$ cells (Supplementary Figure S2). This signal was maintained after $3 \mathrm{~h}$ (Figure 2c). Interestingly, in D492 ${ }^{\mathrm{HER} 2}$ cells the response to EGF treatment was much less pronounced, presumably in part due to the reduced EGFR levels. Phosphorylation of $Y 1068$ showed minimal change, and $Y 1173$ phosphorylation returned to baseline levels within $3 \mathrm{~h}$. Phosphorylation of HER2 could be detected in HER2-expressing cells, and did not respond to EGF treatment. These data demonstrate that HER2 overexpression in D492 made the cells EGF independent and nonresponsive to EGF.

\section{HER2-induced EMT in D492 cells}

The effect of EGFR and HER2 overexpression on the cellular differentiation status was evaluated by measuring the expression of epithelial (CK14, CK19, E-cadherin, p63, P-cadherin) and mesenchymal ( $\mathrm{N}$-cadherin and $\mathrm{Axl}$ ) markers in the transduced cell lines. D492 ${ }^{\mathrm{HER} 2}$ cells showed a near complete loss of CK14 and CK19, E, and P-cadherin and p63 (Figure 3a, upper right), while partial re-expression of the four markers was seen in D492 ${ }^{\text {HER2/EGFR }}$ (Figure 3a, lower right). D492 ${ }^{\mathrm{EGFR}}$ showed a heterogeneous expression pattern of CK19 and CK14, comparable to the control cell line, with most cells being either CK19 positive or CK14 positive while a smaller subpopulation was double positive (Figure 3a, lower left). In D492 ${ }^{\mathrm{EGFR}}$ no change in E-cadherin expression was seen, but expression of P-cadherin was increased, suggesting increased myoepithelial differentiation. The overall expression of luminal-, myoepithelial and mesenchymal markers in the cell lines was also compared using western blotting (Figure 3b). The loss of epithelial markers was confirmed in both $\mathrm{D} 492^{\mathrm{HER} 2}$ and D492 ${ }^{\mathrm{HER} 2 / E G F R}$ as evidenced by reduced expression of P63, CK14 and CK19 as well as E- and P-cadherins. Furthermore, these cells showed increased mesenchymal marker expression as shown by immunostaining with $\mathrm{N}$-cadherin and $\mathrm{Axl}$, indicating that HER2-overexpressing D492 cells have switched to a more mesenchymal phenotype (Figure 3b). D492 ${ }^{\mathrm{EGFR}}$, on the other hand, showed a more pronounced basal/myoepithelial differentiation, as evidenced by stronger CK14, P63 and P-cadherin expression and loss of CK19 (Figure 3b). It is well known that the miR-200 family regulates epithelial morphogenesis and EMT through a negative feedback loop with the ZEB1 and ZEB2 transcription factors. ${ }^{11}$ Compared with $\mathrm{D} 492^{\mathrm{ctrl}}$, the miR-200cmiR141 cluster was greatly downregulated in D492 ${ }^{\text {HER2 }}$ cells (Figure 3c), accompanied with upregulation of ZEB1. Constitutive expression of EGFR, on the other hand, with or without HER2 resulted in increased miR-200-141 expression levels, and reduced
ZEB1 (Figure 3c). Collectively, the results indicated that the tyrosine kinases EGFR and HER2 had distinct effects on differentiation in the breast epithelial progenitor cell line D492. Introduction of HER2 caused a loss of EGFR, cytokeratins and E-cadherin, and increased expression of mesenchymal markers indicating that the cells underwent EMT.

\section{Expression of HER2 leads to non-adherent growth}

D492 ${ }^{\text {ctrl }}$ cells are growth inhibited when they reach confluency in two-dimensional culture. EGFR overexpression affected the growth pattern only to a limited degree (Figure 4a), while D492 $2^{\text {HER2 }}$ and D492 ${ }^{\text {HER2/EGFR }}$ cells had lost their contact inhibition and continued to proliferate after reaching confluency, piling up in culture. (Figure 4a). Increased fraction of non-viable cells was not detected, and all sublines exhibited $95 \%$ and higher viability after 12 days in culture. Furthermore, as colonies of viable cells could be seen floating in the culture medium, we seeded cells into low-attachment plates and quantified survival based on colony formation (Figure 4b). In this environment, a subset of D492 cells was able to survive and proliferate, forming small spheroid clusters (Figure 4b, GFP images). D492 ${ }^{\mathrm{EGFR}}$ did not have significantly higher number of spheroids formed compared with D492 ${ }^{\text {ctrl }}$, but the size of the spheres was significantly increased. D492 ${ }^{\text {HER2 }}$ and D492 ${ }^{\text {HER2/EGFR }}$ showed greatly increased sphere formation. In addition, they were not spherical with intact cell-cell adhesion as seen in D492 ${ }^{\text {ctrl }}$ and D492 ${ }^{\mathrm{EGFR}}$ cells, instead the spheres appeared to form grape-like aggregates, perhaps indicative of changed cell-cell adhesion properties (Figure $4 \mathrm{~b}$, bottom GFP images).

HER2 maintained a mesenchymal phenotype in 3D culture that was partially restored by re-expression of EGFR

Considering the effects of HER2 overexpression on loss of contact inhibition and epithelial markers, it was interesting to compare the phenotypes of all four cell lines when cultured in rBM. The three cell lines that expressed EGFR (ctrl, EGFR and HER2/EGFR) predominantly formed structures with a solid epithelial phenotype with robust cell-cell adherence and some budding/branching structures (Figure 5a). To test the differentiation status of the different 3D phenotypes, immunostaining showed that budding/ branching and round structures expressed epithelial markers, while grape like and spindle did not (Figure 5a). D492 ${ }^{\text {HER2 }}$ cells in rBM showed a diffuse phenotype containing almost only grape- or spindle-like structures. To see whether HER2 receptor signaling was crucial for formation of these mesenchymal colony-type structures, cultures were treated with the HER2 inhibitor CP724,714 (CP). Spindle colony formation was completely inhibited in all cultures when CP treated (Figure $5 \mathrm{a}$, table panel). Furthermore, CP-treated D492 ${ }^{\text {HER2 }}$ cells formed more solid round spheres. These results indicated that HER2 expression is essential for the mesenchymal phenotype to appear, and that EGFR had a stronger effect on morphology in $3 \mathrm{D}$ rBM than in monolayer. Western blotting of lysates from 3D rBM cultures was performed to check whether the EGFR induced appearance of epithelial structures in 3D coincided with re-expression of epithelial markers. Cytokeratins 14 and 19 and E-cadherin were expressed in all EGFRexpressing cell lines in 3D rBM (Figure 5b). The observation that EGFR expression partially reverses HER2-induced EMT in 3D culture but not in monolayer (compare western blots in Figure $3 b$ with Figure $5 b$ ) emphasizes how vastly different these culture conditions are. On the one hand, cells are grown on $2 \mathrm{D}$ collagen-coated plastic, while in the other, cells are embedded in 3D laminin-rich extracellular matrix. In 2D culture, no $\mathrm{N}$-cadherin staining is seen in control cells, while in 3D culture control cells express $\mathrm{N}$-cadherin, indicating a partial switch in phenotype (Figure $5 \mathrm{~b}$ ). It is increasingly being recognized that signaling and gene expression is regulated differently in $3 D$ 
a
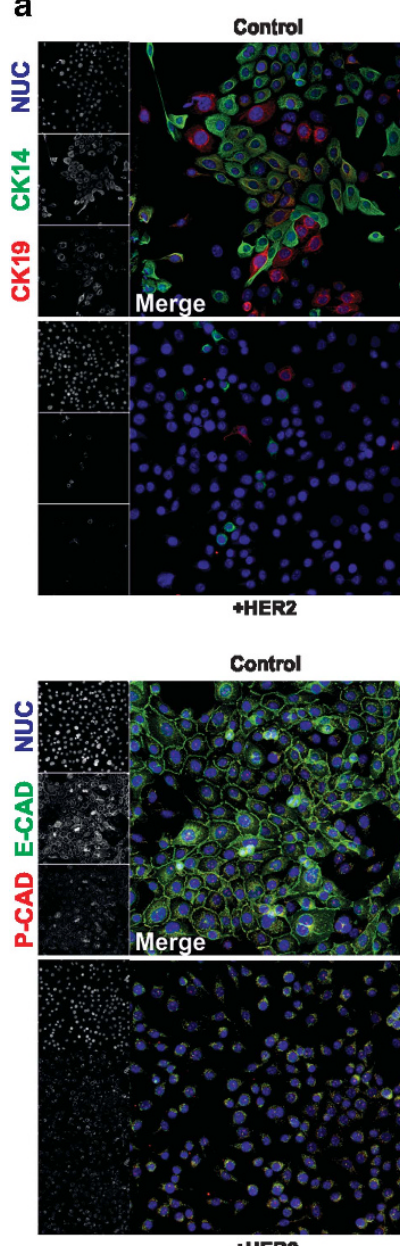

+HER2

Control
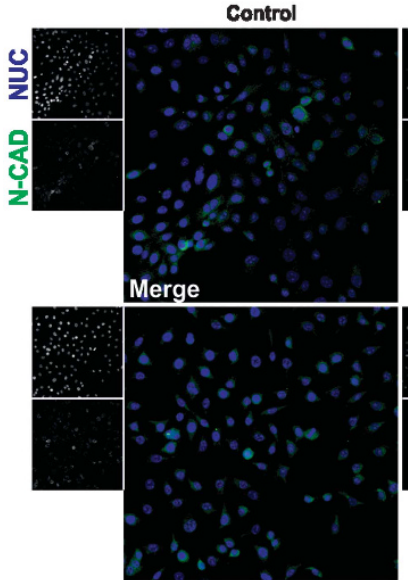

+HER2

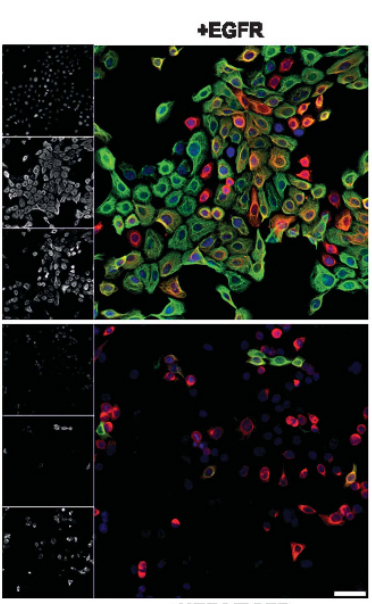

+HER2/EGFR

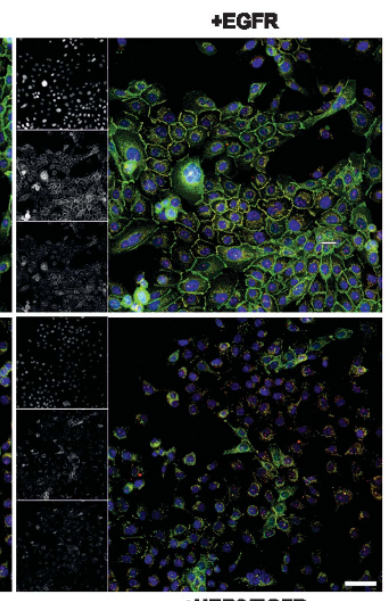

+HER2/EGFR

+EGFR
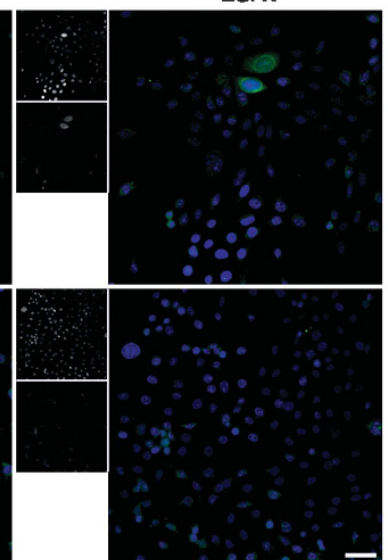

b
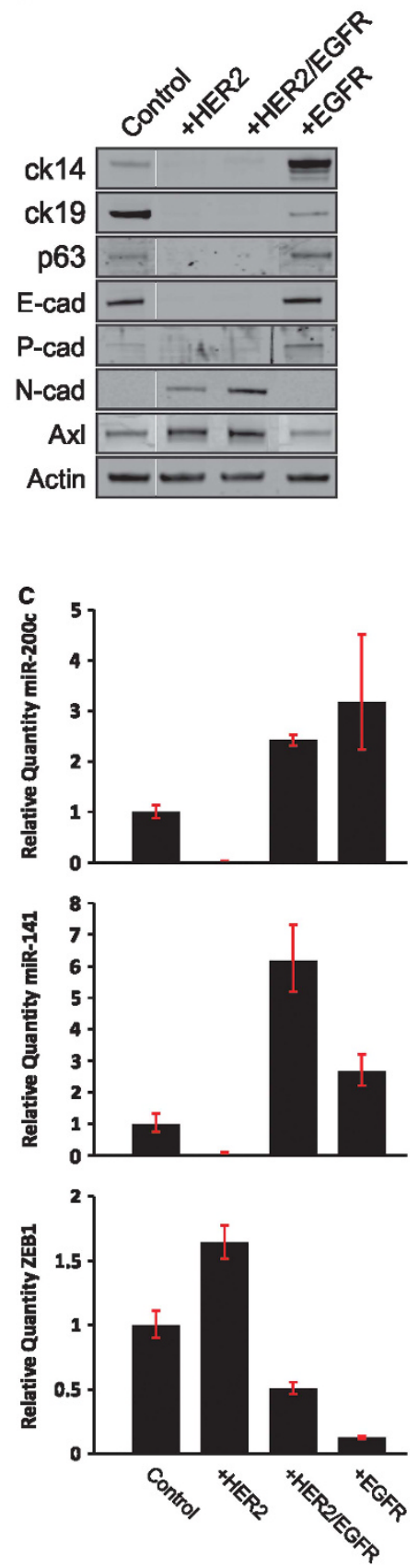

Figure 3. HER2 overexpression induces mesenchymal transition. (a) D492 ${ }^{\text {HER2 }}$ cells lose epithelial phenotype in monolayer culture. Confocal microscopy images of D492 cells expressing ctrl, HER2, EGFR and HER2/EGFR grown on culture flasks and analyzed by immunofluorescence staining for CK14, CK19, E-, N- and P-cadherin expression. Bar $=50 \mu \mathrm{m}$. (b) D492 ${ }^{\mathrm{HER} 2}$ and D492 ${ }^{\mathrm{HER} 2 / \mathrm{EGFR}}$ gain mesenchymal phenotype in monolayer culture. Western blotting performed using lysates from monolayer cultures. Membranes were blotted for CK14, CK19, p63, E-, P-, N-cadherin and Axl expression. Actin = Loading control. (c) In D492 ${ }^{\mathrm{HER} 2}$, miR-200c and miR141 are strongly downregulated. Quantitative reverse transcriptase-PCR was performed using monolayer RNA isolates from all four cell lines. Transcription of miR-200c, miR-141 and ZEB1 was analyzed and normalized to GAPDH.

compared with 2D culture. $^{18}$ These data indicate that EGFR partially inhibits the HER2-induced mesenchymal phenotype with re-appearance of cytokeratins and E-cadherin, in addition to promoting epithelial morphology in 3D cultures.
Ectopic expression of EGFR attenuates HER2-induced tumorigenicity in D492

To test whether EGFR, HER2 or double overexpression of these two receptors affected growth in vivo, the four cell lines were 


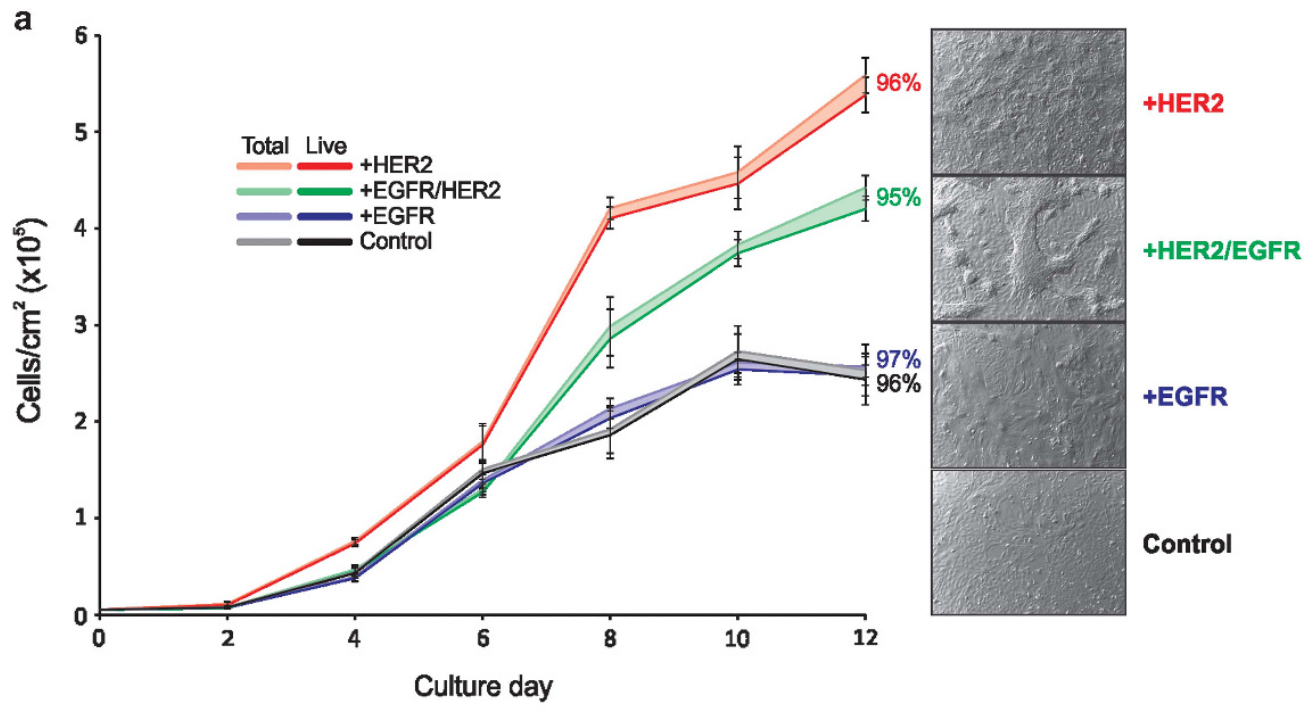

b

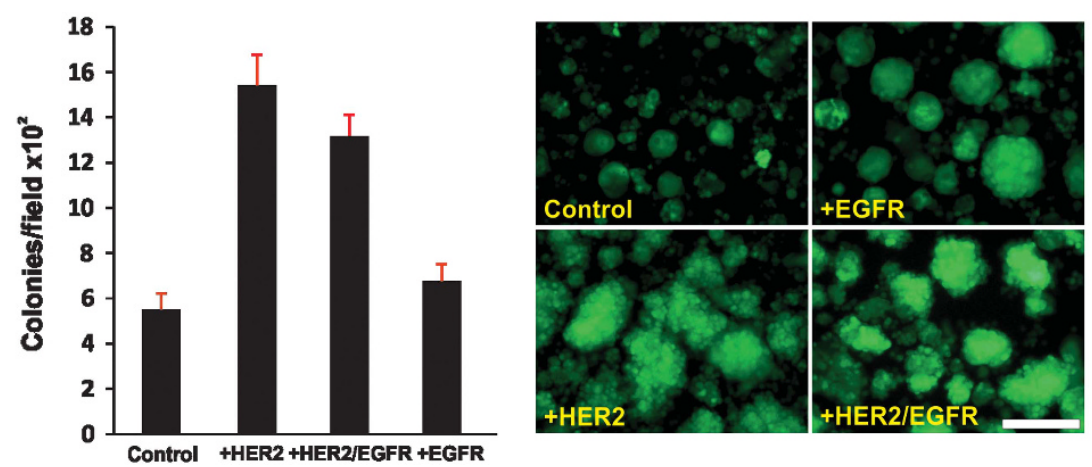

Figure 4. HER2 overexpression leads to loss of growth arrest in monolayer and increased survival in low attachment. (a) D492 ${ }^{\mathrm{HER} 2}$ cells have lost contact inhibition in monolayer culture. D492 cells expressing ctrl, HER2, EGFR and HER2/EGFR were seeded on 24-well plates and cell proliferation measured bi-daily by trypsinizing cells, counting and measuring viability. (b) HER2 leads to higher colony formation in low attachment. D492 cells expressing ctrl, HER2, EGFR and HER2/EGFR were seeded on low-attachment plates and colonies were counted. Bar $=200 \mu \mathrm{m}$.

injected into mammary fat pads of NSG mice. With variable efficiency, all cell lines gave rise to small but palpable tumors 1 week post injection (Figure 6a). HER2 overexpression gave rise to fast growing tumors, while tumors from D492 ${ }^{\text {ctrl }}$ and D492 ${ }^{\text {EGFR }}$ ceased to grow (Figure 6b). Interestingly, and in good concordance with our 3D data, D492 ${ }^{\text {HER2/EGFR }}$ tumors had a longer lag period, and did not grow as fast as D492 ${ }^{\mathrm{HER} 2}$, indicating that EGFR expression suppressed the growth-promoting effect of HER2 (Figure $6 \mathrm{~b}$ and Supplementary Figure S2A). Based on these findings it was interesting to test whether inhibition of EGFR signaling in HER2-expressing cells would affect tumor growth. D492 ${ }^{\text {HER2 }}$ tumor-bearing animals were treated with trastuzumab (HER2 mAB/Herceptin), cetuximab (EGFR mAb/Erbitux) or a combination of the two, and changes in tumor volume were monitored. Upon trastuzumab treatment, tumors decreased significantly in size compared with control, and although some of the individual tumors seemed to regain growth after a lag period, trastuzumab had a clear growth inhibitory effect (Supplementary Figure S4). Interestingly, when administered in combination with cetuximab, the effect of the anti-HER2 mab was less pronounced, as the tumors in this group had a shorter lag period and after 30 days of treatment, they grew equally fast as the control tumors, suggesting that inhibition of EGFR generated faster growth and/or reduced the sensitivity to trastuzumab (Figure $6 c$ ). Cetuximab alone induced a brief pause in tumor growth but after 20 days the related tumors regained a growth rate comparable to the control tumors. The observed in vivo effects of HER2 overexpression and inhibition made it interesting to investigate the patterns of receptor and marker expression within the xenograft tumors. Sections of immunofluorescentlystained D492 ${ }^{\text {HER2 }}$ and D492 $2^{\text {HER2/EGFR }}$ tumors showed that the in vivo expression of epithelial markers correlated well to the $3 \mathrm{D}$ in vitro phenotype (Figure 7). D492 ${ }^{\text {HER2 }}$ cells expressed HER2 but had low EGFR expression. Furthermore, they were CK14 negative demonstrating lack of myoepithelial differentiation and were also E-cadherin and CK19 negative (Figure 7b and Supplementary Figure S3B) In contrast, D492 ${ }^{\text {HER2/EGFR }}$ formed tumors that were heterogeneous in terms of marker expression. These tumors were positive for both HER2 and EGFR with HER2 expressed in all tumor cells, whereas EGFR was partially lost. CK14 expression was restricted to EGFR-expressing cells, thus indicating retention of epithelial characteristics within these tumors (Figure 7b). Additionally, E-cadherin and CK19 staining were seen in EGFRexpressing cells (Figure 7 and Supplementary Figures S3 and S5). Ki67 staining in HER2 and HER2/EGFR tumors was comparable, but markedly higher than in control or EGFR tumors (Figure 7c and Supplementary Figure S3B, respectively). Interestingly, staining for cleaved caspase- 3 indicated that apoptosis levels were considerably higher in HER2/EGFR tumors compared with HER2 alone. Incidentally, active caspase-3 staining was found to be strongest in close proximity to tumor cells with an epithelial phenotype (CK14positive cells; Figure 7c). Staining for cleaved caspase-3 was 
a
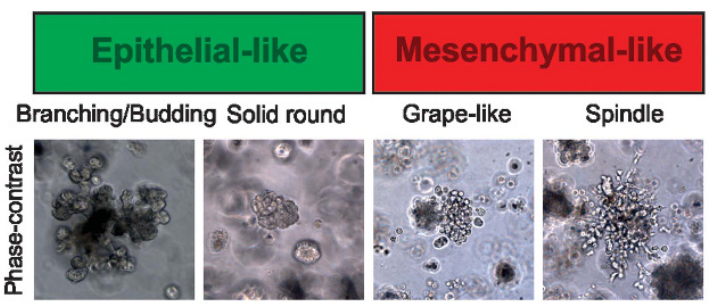

Grape-like

Spindle
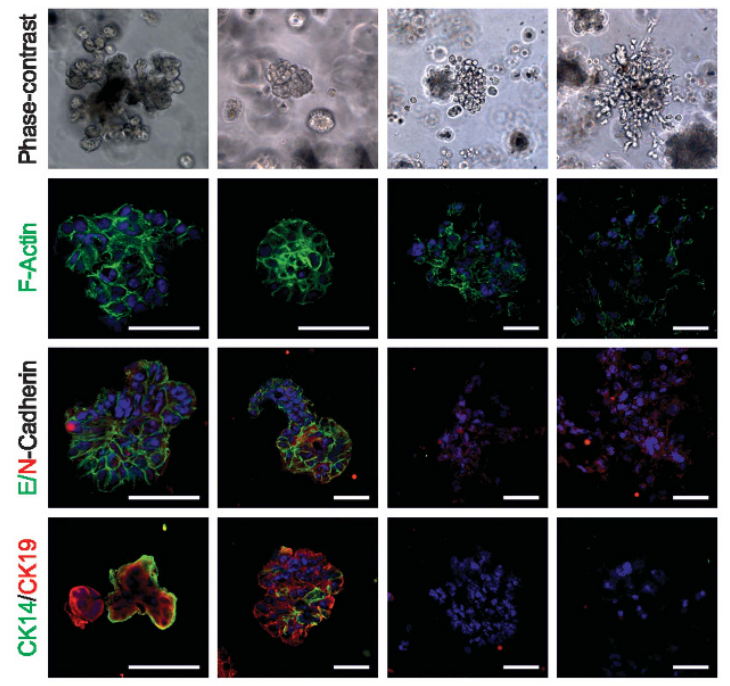

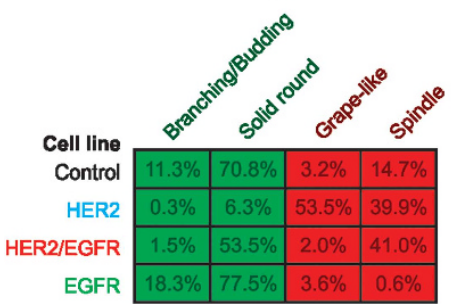

Control

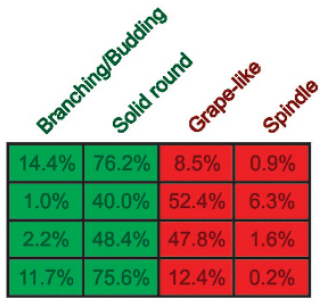

HER2 inhibition
The numbers show the ratio of each 3D phenotype. Left table shows phenotypic ratios in control cultures. Right table shows phenotypic ratios with HER2 inhibitor present

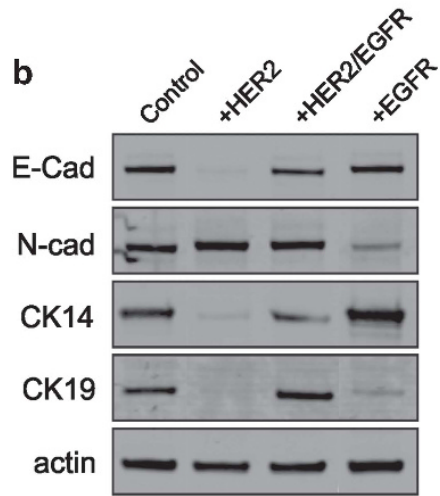

Figure 5. EGFR partially reverses HER2-induced EMT in 3D culture. (a) D492 ${ }^{\mathrm{HER} 2}$ cells show disrupted branching morphogenesis and generate spindle- and grape-like colonies in 3D culture. D492 cells expressing ctrl, HER2, EGFR and HER2/EGFR were seeded into rBM and cultured for 2 weeks. Top picture panel shows representative bright-field images of branching structures found in 3D organotypic cultures of D492 cells. Bar $=100 \mu \mathrm{m}$. Representative isolated structures were fixed and stained for $\mathrm{E}$ - and N-cadherin, CK14 and CK19. Bar $=50 \mu \mathrm{m}$. After the culture period, colonies were counted into four groups, branching/budding, solid round, grape like and spindle. The left table demonstrates the frequencies of each of these phenotypic groups within each D492 subline. The right table demonstrates the same ratios when cultures were treated with the small-molecule HER2 inhibitor CP724,714. (b) EGFR restores the epithelial phenotype in 3D culture in D492 ${ }^{\mathrm{HER} 2}$ (D492 ${ }^{\mathrm{HER} 2 / E G F R}$ ). Western immunoblot for E- and N-cadherin, CK14 and Ck19 of protein lysates isolated from 3D cultures after 14 days of cultivation. a

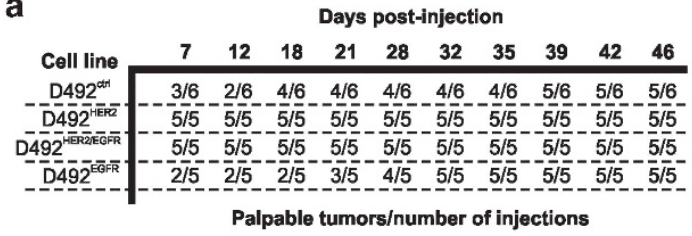

b

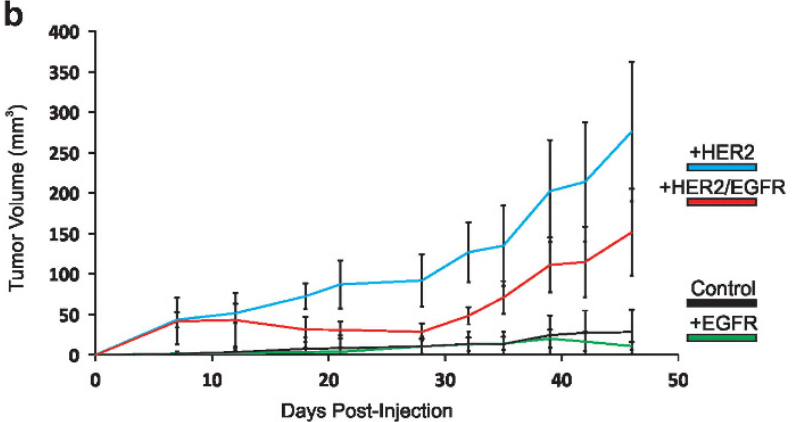

C

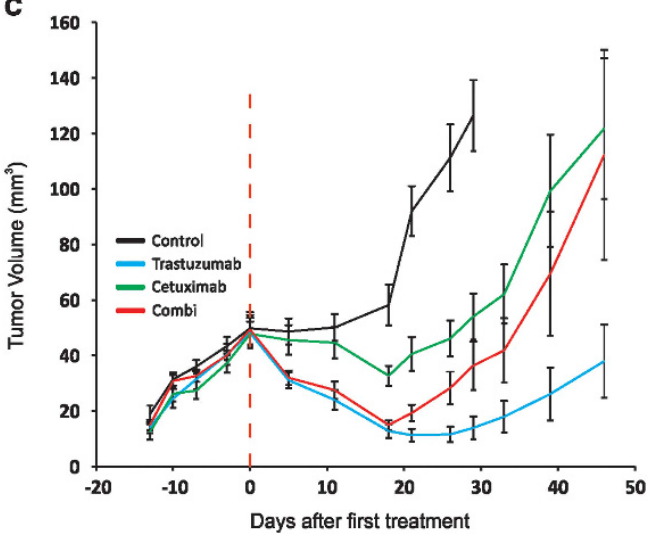

Figure 6. Overexpression of HER2 in D492 leads to increased tumor growth in NSG mice which is partially reversed by EGFR signaling. (a) D492 ${ }^{\mathrm{HER} 2}$ and D492 ${ }^{\mathrm{HER} 2 / E G F R}$ generate tumors efficiently in NSG mice. D492 cells expressing ctrl, HER2, EGFR and HER2/EGFR were injected into NSG mouse mammary fat pads. Numbers represent appearance palpable tumors over total number over injections performed during the course of the experiment. (b) EGFR negatively affects HER2-induced tumor growth. Changes to tumor volumes of D492 cell lines. (c) Cetuximab reduces trastuzumab sensitivity in D492 ${ }^{\text {HER2 }}$ tumors. D492 ${ }^{\text {HER2 }}$ cells were allowed to form tumors in NSG mice. After 2 weeks, mice were randomized into four groups and treated with trastuzumab, cetuximab and a combination of both drugs. Changes to tumor volumes were measured two times weekly.

limited in control and EGFR tumors (Supplementary Figure S3B), indicating low levels of apoptosis.

Collectively, these data showed that coexpression of EGFR and HER2 resulted in slower tumor growth than HER2 alone and suggested that EGFR might suppress the oncogenic properties of HER2, through maintenance of the epithelial phenotype.

\section{DISCUSSION}

Overexpressing two oncogenes, HER2 and EGFR, in the D492 breast epithelial progenitor cell line resulted in changed cellular phenotypes. D492 ${ }^{\mathrm{HER} 2}$ underwent EMT as evidenced by loss of epithelial markers, and gain of mesenchymal markers accompanied by formation of spindle-like colonies in 3D rBM culture. Furthermore, D492 $2^{\mathrm{HER} 2}$ cells showed tumorigenic properties in vivo. These findings are in concordance with other studies where loss of cytokeratins and epithelial cadherins following HER2 
a

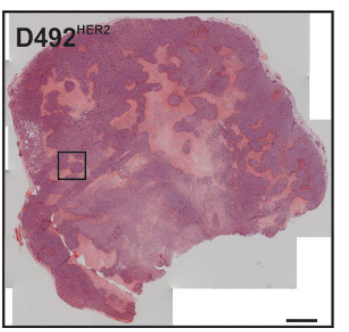

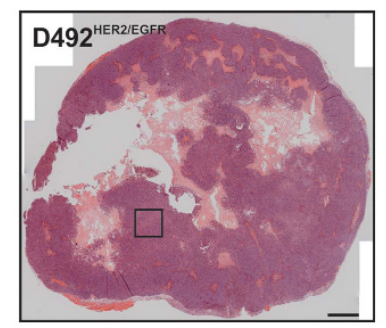

b
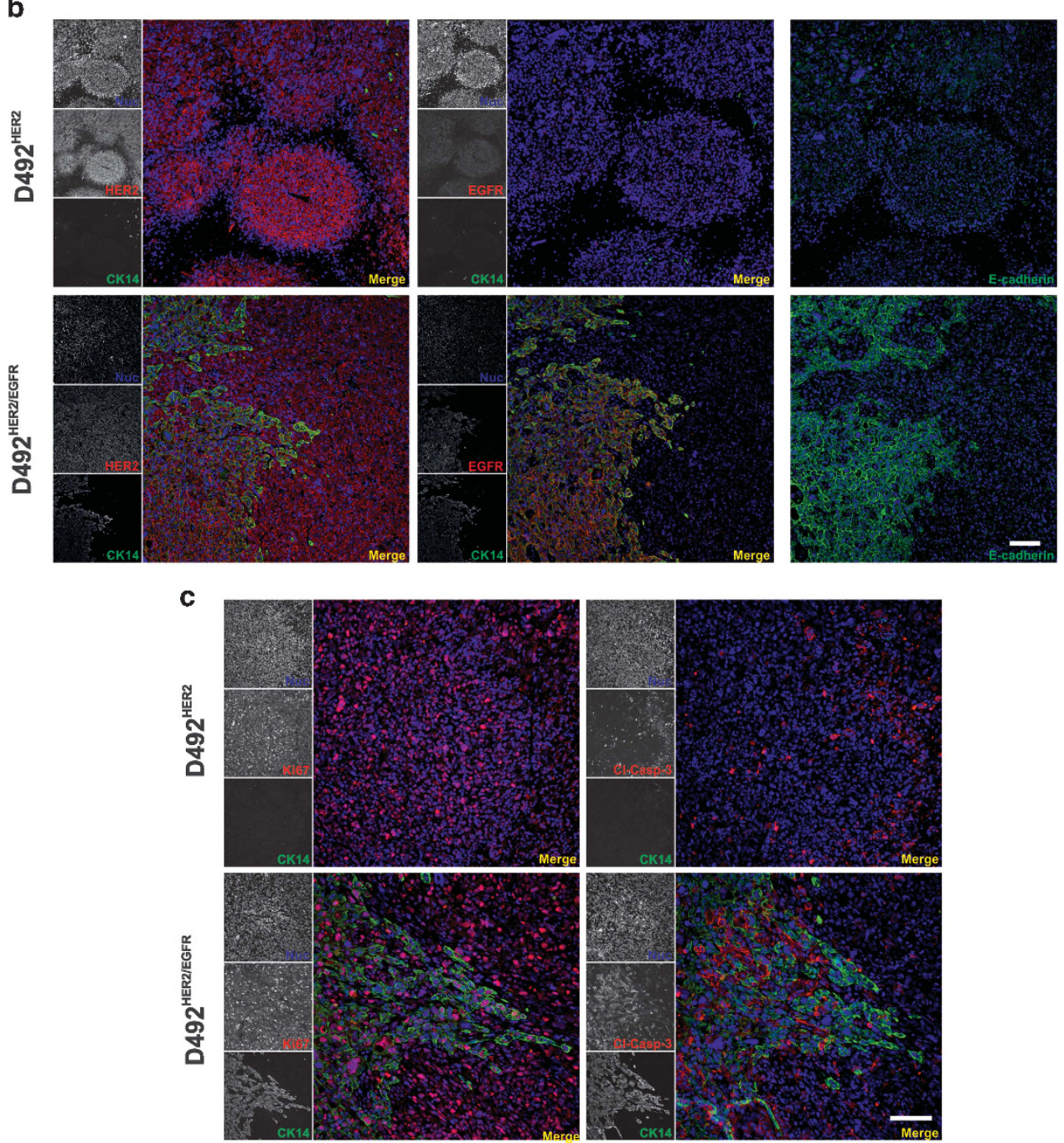

Figure 7. EGFR expression reverses HER2-induced loss of cytokeratin expression in xenografts tumors. (a) Mosaic picture of sections from H\&E staining of D492 ${ }^{\text {HER2 }}$ and D492 ${ }^{\text {HER2/EGFR }}$ tumors. Tumors were paraffin embedded, sliced and stained with H\&E. Squares denote areas represented in immunofluorescence in (b). Bar $=1 \mathrm{~mm}$. (b) EGFR reverses HER2-induced loss of cytokeratin expression. Confocal images showing xenograft slices from D492 ${ }^{\mathrm{HER} 2}$ and D492 ${ }^{\mathrm{HER} 2 / \mathrm{EGFR}}$ tumors stained with HER2, EGFR, CK14 and E-cadherin. Bar $=100 \mu \mathrm{m}$. (c) Apoptosis levels are higher in close proximity to epithelial, CK14-expressing tumor areas. Confocal images showing xenograft slices from D492 ${ }^{\mathrm{HER} 2}$ and D492 ${ }^{\text {HER2/EGRR }}$ tumors stained with CK14, Ki67 and cleaved caspase-3 antibodies. Bar $=100 \mu \mathrm{m}$.

overexpression has been described. ${ }^{19-21}$ HER2 has previously also been overexpressed in non-tumorigenic mammary epithelial cells. $^{22,23}$ In the present study we confirmed that HER2 activity was necessary for the tumor-initiating ability and that simultaneous expression of EGFR suppresses the oncogenic activity of HER2 by maintaining epithelial integrity.

There are a number of studies showing that myoepithelial cells can act as tumor suppressors in the breast gland (reviewed by Barsky et $a l .{ }^{24}$ ). Myoepithelial cells maintain the correct polarity of luminal epithelial cells by providing basement membrane proteins such as laminins. ${ }^{5}$ In cancer, the transition from ductal carcinoma in situ to invasive carcinoma is among other things characterized by the progressive loss of myoepithelial cells. ${ }^{25}$ Forster et al. have recently shown that p63 positive myoepithelial cells are important regulators of epithelial integrity in the breast gland. Loss of p63 resulted in failure of luminal epithelial cells to lactate, due to a failure of myoepithelial cells to express the EGF family member neuregulin. ${ }^{26}$ Interestingly, HER2 overexpression in our model 
prompted a clear decline in EGFR transcription, coinciding with loss of all epithelial markers. One can speculate if the loss of EGFR, which is a driver of myoepithelial differentiation, allowed the more aggressive HER2-expressing cells to proliferate. Supporting this, a loss of EGFR expression accompanied by gain of other EGFR family members during breast cancer progression has been previously described, ${ }^{27}$ and predicted to be a response to changes in HER receptor family downstream signaling, such as estrogen receptor (ER). Indeed, ER status negatively correlates with EGFR expression, as EGFR is often highly expressed in TNBC, ${ }^{28}$ while HER2 is positively correlated with ER. ${ }^{29}$ Our data might indicate that HER2, but not ER, is affecting EGFR expression and phosphorylation. In a recent paper, Wheeler et al. analyzed the correlation between total EGFR, pY1068EGFR expression/phosphorylation and prognosis in head and neck cancer. They demonstrated that total EGFR and pY1068EGFR were prognostic markers in both early stage detected cancers and cetuximab-treated cancers. Furthermore, they also demonstrated that pY1068 was an independent negative factor even when the total EGFR expression was low. This is partially in line with our data, where D492-overexpressing HER2 or HER2/EGFR show low expression of total EGFR but increased phosphorylation of Y1068EGFR. The mechanisms connecting increased Y1068 phosphorylation and reduced tumorigenicity in EGFR/HER2-expressing D492 cells, however, remain to be elucidated.

Although total EGFR expression is lowered we see increased relative phosphorylation levels of Y1068EGFR. EGFR is frequently ubiquitinated and sorted for intracellular degradation. ${ }^{30,31}$ Unlike EGFR, HER2 largely evades ubiquitination, and undergoes internalization and recycling, which prolongs signals generated by HER2-containing heterodimers. ${ }^{32,33}$ Another mechanism that enables HER2 to enhance signaling, despite its inability to directly bind a ligand growth factor, includes inhibition of EGF dissociation from HER2-containing heterodimers. ${ }^{34-36}$

The importance of EGFR expression for preserving the epithelial phenotype was underscored in the experiments where EGFR was re-introduced in D492 ${ }^{\text {HER2 }}$ (D492 ${ }^{\text {HER2/EGFR }}$ ). The in vivo experiments showed that D492 ${ }^{\text {HER2/EGFR }}$ tumors grew slower than D492 ${ }^{\text {HER2 }}$ and the resulting tumors were heterogeneous with a mixed epithelial and mesenchymal phenotype, data that were well supported in the 3D organotypic culture experiments. In addition, treatment of D492 ${ }^{\text {HER2 }}$ xenografts with HER2 and EGFR monoclonal antibodies (trastuzumab and cetuximab, respectively) showed that inhibition of the small EGFR activity found in HER2 xenografts reduced the efficacy of trastuzumab. These data indicated that when expressed in conjunction with HER2, EGFR might act as a tumor suppressor by preserving epithelial differentiation. This result is contrary to what was previously found in xenografts from BT20 mammary epithelial cells overexpressing HER2, where downregulation of EGFR slowed tumor growth. ${ }^{23}$ The importance of context when investigating HER2-induced effects was emphasized by the differences between 2D and 3D culture. In 2D (monolayer) of

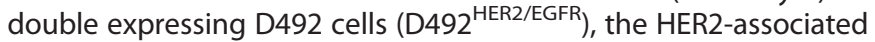
phenotype was dominant over the EGFR phenotype, as evidenced by complete loss of epithelial markers. Interestingly, in 3D assays (and in vivo), EGFR partially reversed the EMT phenotype caused by HER2. These results suggest that 3D organotypic cultures are excellent mimickers of HER2 interactions in vivo, and that microenvironmental context is crucial for what effects HER2 overexpression have on the cellular phenotype.

Understanding the lineage relationship between luminal- and myoepithelial cells in the human breast gland is important to be able to recognize how the normal gland is maintained, how the different subgroups of breast cancer occur and how different clinical outcomes are related to the particular phenotype. In this study, it has been shown that HER2 and EGFR are expressed in luminal- and myoepithelial cells, respectively. In the HER2 subtype of breast cancer, the ErbB2 gene is amplified; expression (although not amplified) of HER2 can also be found in other subtypes such as luminal B and even TNBC. ${ }^{37}$ Balk-Møller and colleagues $^{38}$ have recently demonstrated that HER2-rich tumors and luminal tumors likely share a common origin. This correlates well with the fact that HER2 was predominately expressed in the luminal compartment of human mammary epithelial structures. A large number of luminal B tumors express HER2. Luminal B tumors, although positive for $E R$, do not respond to tamoxifen treatment and have a worse prognosis than luminal A tumors. ${ }^{39}$ The D492 cell line originated from suprabasal cells has a basal-like expression pattern and is not dependent on estrogen, but is highly dependent on EGF for growth. EGFR, along with CK5/6 are the key markers used to identify basal-like breast cancer within TNBC. Basal-like breast cancers, and especially the claudin-low subtype, have been associated with the mesenchymal phenotype. ${ }^{40}$ The EMT program has been associated with many invasive cancer types. This process is seen as an escape pathway for cancer cells to invade the adjacent tissue and subsequently migrate into the lymphatic or blood system. ${ }^{41,42}$ It is, however, important to discriminate between partial EMT where cells retain large part of their epithelial properties and complete EMT where epithelial cells lose their epithelial properties and adopt a complete mesenchymal differentiation state. The latter is one of the hallmarks of cancer. ${ }^{43}$ Interestingly, HER2 overexpression clearly led to a complete EMT with loss of all epithelial markers, downregulation of miR-200c-141 and increased expression of ZEB1. Tumor cells with a mesenchymal phenotype are described as highly aggressive and more resistant to treatment than other cancer cell populations. ${ }^{44,45}$ In the event that HER2 is the initiator of the aggressive mesenchymal phenotype, therapy regimes might become more effective and less exposed to resistance if they are designed to both inhibit HER2 and the mesenchymal phenotype. Interestingly, even a very low expression of EGFR and low EGFR activity was sufficient to counteract HER2-induced EMT and reintroduce cytokeratin expression in 3D culture and in vivo. The signaling events upheld by EGFR might represent attractive possibilities for anti-HER2 and anti-EMT therapy.

In this work, we have explored the roles of HER2 and EGFR in mammary progenitor cells. The data introduced a novel role for EGFR in breast morphogenesis and cancer; if expressed in a defined context with HER2, EGFR may act as a tumor suppressor by maintaining epithelial integrity. These data indicate that in certain tumors, where EGFR expression is present, inhibiting the receptor might lead to changes in marker expression and loss of epithelial characteristics, emphasizing the importance of context when devising treatment strategies.

\section{MATERIALS AND METHODS}

Cell culture

D492 cells were maintained in $\mathrm{H} 14$ media as described previously, ${ }^{17}$ in tissue culture treated, collagen I (Advanced Biomatrix, San Diego, CA, USA)coated T25 Falcon flasks (BD Biosciences (BD), Franklin Lakes, NJ, USA). The cells were subcultured weekly in a 1:20 ratio in new flasks. Cells were fed three times per week and routinely checked for mycoplasma contamination

\section{Immunohistochemistry}

Cryosections from fresh-frozen tissue samples from reduction mammoplasties (Icelandic National Bioethics Committee No. VSN-13-057) or 3D experiments were fixed in $3.7 \%$ formaldehyde for $15 \mathrm{~min}$ and permeabilized with $0.1 \%$ Triton-X for 10 min. Samples were blocked using $5 \%$ normal goat serum (Life Technologies, Carlsbad, CA, USA) and incubated with primary antibody overnight at $4{ }^{\circ} \mathrm{C}$. Samples were then stained with isotype specific secondary fluorescent Alexa Fluor goat antibodies (Life Technologies) and counterstained with 4',6-diamidino-2-phenylindole (DAPI) (Sigma-Aldrich, St Louis, MO, USA). Samples were visualized using an Olympus FV1200 Confocal microscope (Olympus, Tokyo, Japan). 
Sections from paraffin-embedded mouse tissue were processed in xylene and ethanol to remove paraffin, and then rehydrated in $\mathrm{dH}_{2} \mathrm{O}$. After rehydration, high-temperature antigen retrieval was performed. Samples were stained as described above.

\section{Antibodies used for immunostaining/western blotting}

The following antibodies were used in this study: CK14 (Leica Microsystems, Wetzlar, Germany, LL-002 and Abcam, Cambridge, UK, ab 15461), CK19 (Abcam, ab7754), Actin (Abcam ab8229), glyceraldehyde 3-phosphate dehydrogenase (Abcam, ab9484), p63 (Abcam, ab3239), E-cadherin (BD, BD610682), N-cadherin (BD, BD610921), P-cadherin (Cell Signaling (CS), Danvers, MA, USA, \#2130), EGFR (CS\#4267), EGFR pY1068 (CS\#3777), EGFR pY1173 (CS\#4407), HER2 (CS\#2165) HER2 pY1221/1222 (CS\#2243), Axl (CS\#8661), Ki67 (Abcam, ab15580), Cleaved Caspase-3 (Abcam, ab2302) and Phalloidin (Life Technologies, A22283, A12379).

\section{Viral transduction}

All vectors used for viral production or cloning were acquired from Addgene and are shown in Supplementary Figure S1. pBABE-EGFR and empty backbone (\#11011, \#1764, respectively ${ }^{46}$ ) were used as provided. HER2 coding sequence $\left(\# 16257^{47}\right)$ was cloned into the lentiviral vector pWPI (\#12254, gift from Didier Trono). Retroviral (EGFR) virus production was performed in Phoenix HEK293 cells, using Arrestin transfection (Life Technologies). D492 cells were infected overnight with viral supernatant containing $8 \mu \mathrm{g} / \mathrm{ml}$ Polybrene (Sigma-Aldrich). EGFRtransduced cells were selected using $2 \mu \mathrm{g} / \mathrm{ml}$ puromycin (Sigma-Aldrich). HER2-containing lentiviral plasmids were transfected into HEK293T cells using Arrestln, and D492 cells were infected with viral supernatant containing $8 \mu \mathrm{g} / \mathrm{ml}$ polybrene. HER2-overexpressing cells were selected based on eGFP expression in FACSaria (BD). Control cells were transduced with both empty vector backbones for EGFR and HER2.

\section{Primary cultures}

For western blotting we isolated normal primary mammary cells from reduction mammoplasties. Donor material was given after acquiring informed consent from the donor. Approved by the Icelandic National Bioethics Committee VSN-13-057.

Primary normal human mammary tissue was processed as previously described. ${ }^{48}$ Luminal epithelial cells were sorted by positive selection or MUC1 (Life Technologies) and EpCAM (Leica) using magnetic cell sorting (Miltenyi Biotech, Bergisch Gladbach, Germany), whereas myoepithelial cells were selected for using Thy-1 (Dianova, Hamburg, Germany) and subsequently with beta 4 integrin (Millipore, Billerica, MA, USA) selection.

\section{D assays}

For 3D assays, $10^{4}$ cells were seeded into $300 \mu \mathrm{l}$ Matrigel (BD) and maintained over 15 days. Culture media $(\mathrm{H} 14)$ was changed three times per week. After the culture period, cultures were processed for protein extraction or immunostaining of isolated colonies as described by Lee et al. ${ }^{49}$ For experiments when cells were treated with the HER2 inhibitor CP724,714, media was supplemented with a final concentration of $100 \mathrm{nM}$ inhibitor dissolved in dimethyl sulfoxide.

\section{Proliferation assay}

Cells were seeded in triplicate into collagen-coated six-well trays at a density of 50000 cells per well in H14 media. Every other day, cells were detached and combined with the floating fraction from the spent media. Cells were then stained with Acridine Orange and DAPI (both from Chemometec A/S, Allerod, Denmark): staining all cells and dead cells, respectively. Viable and dead cells were counted using NucleoCounter3000 (Chemometec)

\section{Western blotting}

Protein lysates were acquired using RIPA lysis buffer supplemented with both phosphatase and protease inhibitor cocktails (Life Technologies). For western blots, $5 \mu \mathrm{g}$ protein was used per lane, unless otherwise stated. Samples were denatured using $10 \%$ mercaptoethanol at $95^{\circ} \mathrm{C}$ for $5 \mathrm{~min}$ and run on NuPage 10\% Bis-Tris gels (Life Technologies) in 2-(N-morpholino)ethanesulfonic acid (MES) running buffer. Samples were then transferred to Immobilon FL PVDF membranes (Millipore). Membranes were blocked in Li-cor blocking buffer and primary antibodies were incubated overnight at $4^{\circ} \mathrm{C}$. Near-infrared fluorescence visualization was measured using Odyssey CLx scanner (Li-Cor, Cambridge, UK).

\section{Low attachment assay}

For low-attachment assays, six-well trays were coated with $1.2 \mathrm{mg} / \mathrm{ml}$ polyHEMA (Sigma-Aldrich) overnight at $37^{\circ} \mathrm{C}$. Cells were seeded in triplicate at a density of $10^{3}$ per well, and cultured for 2 weeks. After the culture period, colonies were imaged and counted.

\section{Quantitative reverse transcriptase-PCR}

Total RNA was extracted with Trizol (Life Technologies), DNAase treated and reverse transcribed with hexanucleotides using ReverAid (\#K1622, Life Technologies). Resulting CDNA was used for quantitative reverse transcriptase-PCR, in master mix (Life Technologies) with primer pairs and probes for EGFR (Hs00540086_m1, Life Technologies), HER2 (Hs01076092_m1), ZEB1 (Hs00232783_m1) and glyceraldehyde 3-phosphate dehydrogenase (Hs99999905_m1). Experiments were carried out in triplicate on 7500 Real Time PCR System (Life Technologies). EGFR, HER2 and ZEB1 mRNA levels were normalized to glyceraldehyde 3-phosphate dehydrogenase and relative mRNA differences were calculated with the $2^{\Delta \mathrm{Ct}}$ method.

Quantitative reverse transcriptase-PCR analysis of miRNAs was performed using miRCURY LNA microRNA PCR System (Exiqon, Copenhagen, Denmark). Gene expression levels were quantified using primers for hsa-miR-141 (\#204504) and hsa-miR-200c (\#2044852) (Exiqon). Normalization was carried out with U6 RNA(\#203907)(Exiqon).

\section{NSG xenografts}

The in vivo tumorigenicity assays were performed using NOD/SCID interleukin-2 receptor gamma chain null (NSG $\| 2 \mathrm{rg}-/-$ ) mice, under unblinded conditions. The animals $(n=23)$ were randomized into four groups according to size, and $5 \times 10^{5}$ cells were injected bilaterally in $100 \mu \mathrm{l}$ phosphate-buffered saline/matrigel. Group sizes were as follows: $\mathrm{D} 492^{\mathrm{ctrl}}, n=5 ; \mathrm{D} 492^{\mathrm{EGFR}}, \mathrm{D} 492^{\mathrm{HER} 2}$ and D492 $2^{\mathrm{HER} 2 / \mathrm{EGFR}}, n=6$ for each group. Distilled tap water was given ad libitum, supplemented with $17-\beta$-estradiol at a concentration of $4 \mathrm{mg} / \mathrm{l}$. For drug experiments, 23 mice were injected bilaterally with $5 \times 10^{5}$ D492 ${ }^{\text {HER2 }}$ cells. Mice were treated intraperitoneally with $4 \mathrm{mg} / \mathrm{kg}$ trastuzumab, $20 \mathrm{mg} / \mathrm{kg}$ cetuximab or a combination of the two ( $n=6$ for each group, $n=5$ for control mice) from day 0 ( 13 days post injection), once a week on days $0,7,14$ and 21 . All mice used in the experiment were locally bred at the Department of Comparative Medicine (Oslo University Hospital, Oslo, Norway). All procedures and experiments involving animals were approved by the National Animal Research Authority (http://www.fdu.no/). The experiments were conducted according to the regulations of the Federation of European Laboratory Animal Science Association (FELASA), and performed according to the $3 \mathrm{R}$ principle (Replacement, Reduction, and Refinement). Mice were killed before the end of the experiment if tumor size exceeded $20 \mathrm{~mm}$ diameter, or if mice appeared unhealthy or in pain. Tumors were measured two times weekly, and after the experimental period, tumors were harvested, formalin fixed and embedded in paraffin.

Statistical analysis

All experiments were performed at minimum in triplicate. Graphs were generated in Microsoft Excel. Error bars represent the standard deviation of the mean.

\section{CONFLICT OF INTEREST}

The authors declare no conflict of interest.

\section{ACKNOWLEDGEMENTS}

This work was supported from Landspitali University Hospital Science Fund, University of Iceland Research Fund, Science and Technology Policy Council Research Fund and Grant of Excellence, 'Göngum saman', a supporting group for breast cancer research in Iceland. The funders had no role in study design, data collection and 
analysis, decision to publish or preparation of the manuscript. We are grateful to Petros Gebregziabher and Alexandr Kristian for advicing on and performing the in vivo experiments.

\section{REFERENCES}

1 Perou CM, Sorlie T, Eisen MB, van de Rijn M, Jeffrey SS, Rees CA et al. Molecular portraits of human breast tumours. Nature 2000; 406: 747-752.

2 Sorlie T, Perou CM, Tibshirani R, Aas T, Geisler S, Johnsen H et al. Gene expression patterns of breast carcinomas distinguish tumor subclasses with clinical implications. Proc Natl Acad Sci USA 2001; 98: 10869-10874.

3 Visvader JE, Stingl J. Mammary stem cells and the differentiation hierarchy: current status and perspectives. Genes Dev 2014; 28: 1143-1158.

4 Oakes SR, Gallego-Ortega D, Ormandy CJ. The mammary cellular hierarchy and breast cancer. Cell Mol Life Sci 2014; 71: 4301-4324.

5 Gudjonsson T, Ronnov-Jessen L, Villadsen R, Rank F, Bissell MJ, Petersen OW. Normal and tumor-derived myoepithelial cells differ in their ability to interact with luminal breast epithelial cells for polarity and basement membrane deposition. J Cell Sci 2002; 115(Pt 1): 39-50.

6 Sigurdsson V, Hilmarsdottir B, Sigmundsdottir H, Fridriksdottir AJ, Ringner M, Villadsen R et al. Endothelial induced EMT in breast epithelial cells with stem cell properties. PloS One 2011; 6: e23833.

7 Moyret-Lalle C, Ruiz E, Puisieux A. Epithelial-mesenchymal transition transcription factors and miRNAs: "Plastic surgeons" of breast cancer. World J Clin Oncol 2014; 5: 311-322.

8 Dykxhoorn DM, Wu Y, Xie H, Yu F, Lal A, Petrocca F et al. miR-200 enhances mouse breast cancer cell colonization to form distant metastases. PloS One 2009; 4: e7181.

9 Tam WL, Weinberg RA. The epigenetics of epithelial-mesenchymal plasticity in cancer. Nat Med 2013; 19: 1438-1449.

10 Hilmarsdottir B, Briem E, Sigurdsson V, Franzdottir SR, Ringner M, Arason AJ et al. MicroRNA-200c-141 and Np63 are required for breast epithelial differentiation and branching morphogenesis. Dev Biol 2015; 403: 150-161.

11 Hilmarsdottir B, Briem E, Bergthorsson JT, Magnusson MK, Gudjonsson T. Functional role of the microRNA-200 family in breast morphogenesis and neoplasia. Genes 2014; 5: 804-820.

12 Slamon DJ, Clark GM, Wong SG, Levin WJ, Ullrich A, McGuire WL. Human breast cancer: correlation of relapse and survival with amplification of the HER-2/neu oncogene. Science (New York, NY) 1987; 235: 177-182.

13 Gupta P, Srivastava SK. HER2 mediated de novo production of TGFbeta leads to SNAIL driven epithelial-to-mesenchymal transition and metastasis of breast cancer. Mol Oncol 2014; 8: 1532-1547.

14 Sternlicht MD, Kouros-Mehr H, Lu P, Werb Z. Hormonal and local control of mammary branching morphogenesis. Differentiation 2006; 74: 365-381.

15 Davis NM, Sokolosky M, Stadelman K, Abrams SL, Libra M, Candido S et al. Deregulation of the EGFR/PI3K/PTEN/Akt/mTORC1 pathway in breast cancer: possibilities for therapeutic intervention. Oncotarget 2014; 5: 4603-4650.

16 Guth U, Magaton I, Huang DJ, Fisher R, Schotzau A, Vetter M. Primary and secondary distant metastatic breast cancer: two sides of the same coin. Breast (Edinburgh, Scotland) 2014; 23: 26-32.

17 Gudjonsson T, Villadsen R, Nielsen HL, Ronnov-Jessen L, Bissell MJ, Petersen OW. Isolation, immortalization, and characterization of a human breast epithelial cell line with stem cell properties. Genes Dev 2002; 16: 693-706.

18 Vidi PA, Bissell MJ, Lelievre SA. Three-dimensional culture of human breast epithelial cells: the how and the why. Methods Mol Biol (Clifton, NJ) 2013; 945: 193-219.

19 Ortega-Cava CF, Raja SM, Laiq Z, Bailey TA, Luan H, Mohapatra B et al. Continuous requirement of ErbB2 kinase activity for loss of cell polarity and lumen formation in a novel ErbB2/Neu-driven murine cell line model of metastatic breast cancer. J Carcinog 2011; 10: 29.

20 Jenndahl LE, Isakson P, Baeckstrom D. c-erbB2-induced epithelial-mesenchymal transition in mammary epithelial cells is suppressed by cell-cell contact and initiated prior to E-cadherin downregulation. Int $J$ Oncol 2005; 27: 439-448.

21 Carpenter RL, Paw I, Dewhirst MW, Lo HW. Akt phosphorylates and activates HSF-1 independent of heat shock, leading to Slug overexpression and epithelialmesenchymal transition (EMT) of HER2-overexpressing breast cancer cells. Oncogene 2014; 34: 546-557.

22 Zhan L, Xiang B, Muthuswamy SK. Controlled activation of ErbB1/ErbB2 heterodimers promote invasion of three-dimensional organized epithelia in an ErbB1dependent manner: implications for progression of ErbB2-overexpressing tumors. Cancer Res 2006; 66: 5201-5208.

23 Hartman Z, Zhao H, Agazie YM. HER2 stabilizes EGFR and itself by altering autophosphorylation patterns in a manner that overcomes regulatory mechanisms and promotes proliferative and transformation signaling. Oncogene 2013; 32: 4169-4180.

24 Barsky SH, Karlin NJ. Myoepithelial cells: autocrine and paracrine suppressors of breast cancer progression. J Mammary Gland Biol Neoplasia 2005; 10: 249-260.

25 Hu M, Yao J, Carroll DK, Weremowicz S, Chen H, Carrasco D et al. Regulation of in situ to invasive breast carcinoma transition. Cancer Cell 2008; 13: 394-406.

26 Forster N, Saladi SV, van Bragt M, Sfondouris ME, Jones FE, Li Z et al. Basal cell signaling by $\mathrm{p} 63$ controls luminal progenitor function and lactation via NRG1. Dev Cell 2014; 28: 147-160.

27 Flageng MH, Knappskog S, Haynes BP, Lonning PE, Mellgren G. Inverse regulation of EGFR/HER1 and HER2-4 in normal and malignant human breast tissue. PloS One 2013; 8: e74618.

28 Sobande F, Dusek L, Matejkova A, Rozkos T, Laco J, Ryska A. EGFR in triple negative breast carcinoma: significance of protein expression and high gene copy number. Cesk Patol 2015; 51: 80-86.

29 Pinhel I, Hills M, Drury S, Salter J, Sumo G, A'Hern R et al. ER and HER2 expression are positively correlated in HER2 non-overexpressing breast cancer. Breast Cancer Res 2012; 14: R46.

30 Zwang Y, Yarden Y. Systems biology of growth factor-induced receptor endocytosis. Traffic (Copenhagen, Denmark) 2009; 10: 349-363.

31 Haglund K, Dikic I. The role of ubiquitylation in receptor endocytosis and endosomal sorting. J Cell Sci 2012; 125(Pt 2): 265-275.

32 Pinkas-Kramarski R, Soussan L, Waterman H, Levkowitz G, Alroy I, Klapper L et al. Diversification of Neu differentiation factor and epidermal growth factor signaling by combinatorial receptor interactions. EMBO J 1996; 15: 2452-2467.

33 Baulida J, Kraus MH, Alimandi M, Di Fiore PP, Carpenter G. All ErbB receptors other than the epidermal growth factor receptor are endocytosis impaired. J Biol Chem 1996; 271: 5251-5257.

34 Tzahar E, Waterman H, Chen X, Levkowitz G, Karunagaran D, Lavi S et al. A hierarchical network of interreceptor interactions determines signal transduction by Neu differentiation factor/neuregulin and epidermal growth factor. $\mathrm{Mol}$ Cell Biol 1996; 16: 5276-5287.

35 Graus-Porta D, Beerli RR, Daly JM, Hynes NE. ErbB-2, the preferred heterodimerization partner of all ErbB receptors, is a mediator of lateral signaling. $E M B O$ J 1997; 16: 1647-1655.

36 Schneider MR, Yarden Y. The EGFR-HER2 module: a stem cell approach to understanding a prime target and driver of solid tumors. Oncogene 2016; 35: 2949-2960.

37 Nakagawa M, Bando Y, Nagao T, Takai C, Ohnishi T, Honda J et al. Among triplenegative breast cancers, HER2(0) breast cancer shows a strong tendency to be basal-like compared with HER2(1+) breast cancer: preliminary results. Breast Cancer (Tokyo, Japan) 2012; 19: 54-59.

38 Balk-Moller E, Kim J, Hopkinson B, Timmermans-Wielenga V, Petersen OW, Villadsen R. A marker of endocrine receptor-positive cells, CEACAM6, is shared by two major classes of breast cancer: luminal and HER2-enriched. Am J Pathol 2014; 184: 1198-1208.

39 Zhang MH, Man HT, Zhao XD, Dong N, Ma SL. Estrogen receptor-positive breast cancer molecular signatures and therapeutic potentials (review). Biomed Rep 2014; 2: 41-52.

40 Sabatier R, Finetti P, Guille A, Adelaide J, Chaffanet M, Viens P et al. Claudin-low breast cancers: clinical, pathological, molecular and prognostic characterization. Mol Cancer 2014; 13: 228.

41 Lee K, Gjorevski N, Boghaert E, Radisky DC, Nelson CM. Snail1, Snail2, and E47 promote mammary epithelial branching morphogenesis. EMBO J 2011; 30: 2662-2674.

42 Taube JH, Herschkowitz Jl, Komurov K, Zhou AY, Gupta S, Yang J et al. Core epithelial-to-mesenchymal transition interactome gene-expression signature is associated with claudin-low and metaplastic breast cancer subtypes. Proc Natl Acad Sci USA 2010; 107: 15449-15454.

43 Hanahan D, Weinberg RA. Hallmarks of cancer: the next generation. Cell 2011; 144: 646-674.

44 Hennessy BT, Gonzalez-Angulo AM, Stemke-Hale K, Gilcrease MZ, Krishnamurthy $S$, Lee JS et al. Characterization of a naturally occurring breast cancer subset enriched in epithelial-to-mesenchymal transition and stem cell characteristics. Cancer Res 2009; 69: 4116-4124.

45 Creighton CJ, Li X, Landis M, Dixon JM, Neumeister VM, Sjolund A et al. Residual breast cancers after conventional therapy display mesenchymal as well as tumor-initiating features. Proc Natl Acad Sci USA 2009; 106: 13820-13825.

46 Greulich H, Chen TH, Feng W, Janne PA, Alvarez JV, Zappaterra M et al. Oncogenic transformation by inhibitor-sensitive and -resistant EGFR mutants. PLoS Med 2005; 2: e313. 
47 Li YM, Pan Y, Wei Y, Cheng X, Zhou BP, Tan M et al. Upregulation of CXCR4 is essential for HER2-mediated tumor metastasis. Cancer Cell 2004; 6: 459-469.

48 Ingthorsson S, Sigurdsson V, Fridriksdottir A Jr, Jonasson JG, Kjartansson J, Magnusson MK et al. Endothelial cells stimulate growth of normal and cancerous breast epithelial cells in 3D culture. BMC Res Notes 2010; 3: 184.

49 Lee GY, Kenny PA, Lee EH, Bissell MJ. Three-dimensional culture models of normal and malignant breast epithelial cells. Nat Methods 2007; 4: 359-365. cc) (i) $\Theta$ This work is licensed under a Creative Commons Attributioncc. NonCommercial-NoDerivs 4.0 International License. The images or other third party material in this article are included in the article's Creative Commons license, unless indicated otherwise in the credit line; if the material is not included under the Creative Commons license, users will need to obtain permission from the license holder to reproduce the material. To view a copy of this license, visit http:// creativecommons.org/licenses/by-nc-nd/4.0/

Supplementary Information accompanies this paper on the Oncogene website (http://www.nature.com/onc) 